\title{
Analysis of von Kármán plates using a BEM formulation
}

\author{
L. Waidemam \& W. S. Venturini \\ São Carlos School of Engineering, University of São Paulo, Brazil
}

\begin{abstract}
This work deals with non-linear geometrical plates in the context of von Kármán theory. The formulation is written in a way to require only boundary in-plane displacement and deflection integral equation for boundary collocations. At internal points only out of plane rotation, curvature and in-plane internal force representations are used. The non-linear system of algebraic equations to be solved is reduced to internal point collocation relations. The solution is solved by using a Newton scheme for which a consistent tangent operator was derived. Keywords: bending plates, geometrical nonlinearities.
\end{abstract}

\section{Introduction}

The boundary element method (BEM) applied to solve plate-bending problems has been successfully used many times so far. An important characteristic of the boundary methods applied to plate bending is approximating all boundary values by the same shape function, avoiding therefore using higher order derivatives of displacement approximation to compute internal forces. Thus, bending and twisting moments and also shear forces are precisely evaluated. The method has already proved to be enough accurate and reliable for this kind of application.

The plate bending numerical formulation is a very important subject in engineering due to be applied to a large number of complex problems such as aircraft, ship, car, pressure vessel, off shore structures among others. Usually these complex problems require accurate plate bending models as those that take into account the geometrical non-linear effects. In this context, several BEM formulations have already been proposed so far. One of the first works treating this subject is due to Morjaria [1]. Kamiya and Sawaki [2] have proposed a BEM formulation for elastic plates governed by the Berger equation. The first BEM 
formulation to analyze plate-bending problems within the context of von Kármán hypothesis is due to Ye and Liu [3], who have used a fictitious loading distributed over the domain to model the non-linear effects. Von Kármán hypothesis was also adopted by Tanaka et al [4] to develop a more elaborated BEM incremental formulation to deal with finite deflections of thin elastic plates. Wang et al [5] have also worked on von Kármán plates introducing the dual reciprocity approach based on global radial functions to approximate the correcting integral term.

All works reported above were proposed in the context of thin plates. Several other important works have appeared more recently pointing out the efficiency of BEM formulations to deal with shear deformable plate based on the ReissnerMindlin hypothesis: Wen et al [6], Purbolaksono and Aliabadi [7].

In this work we came back to the BEM formulation based on the von Kármán's theory. Emphasis is given to the accurate evaluation of the domain integrals approximated by using cells and to the solution technique for which a tangent consistent operator is proposed. Examples of plate with finite deflection is analysed and the results compared with other numerical solutions.

\section{Basic equations}

Without loss of generality, let us consider a single thin plate region $\Omega$ with boundary $\Gamma$ over which a distributed load $q$ is applied orthogonal to the middle surface, (direction $x_{3}$ ), as shown in figure 1 . This plate region can also be subjected to in plane forces (directions $x_{1}$ and $x_{2}$ ) either distributed over the domain or applied along the boundary. In order to write the field equations of this plate problems following the hypothesis can be assumed according to the von Kármán theory, for which the strains are assumed to be enough small and the final deflection of order of the plate thickness $h$.

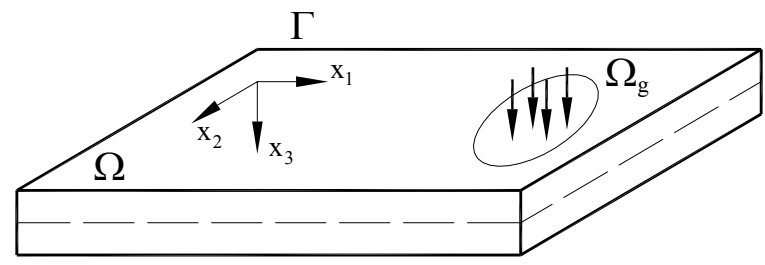

Figure 1: $\quad$ General plate domain.

For any point defined in $\Omega$ the following basic relationships are defined:

- Equilibrium equations for the bending problem:

$$
m_{i j},_{i j}+N_{i j} w_{,_{i j}}-b_{i} w_{,_{i}}+m_{i},_{i}+q=0
$$


where $m_{i j}$ are bending and twisting moments, $N_{i j}$ is the in-plane internal forces, $b_{i}$ represents the in-plane domain loads applied and $m_{i}$ is the applied moment over the plate domain; the subscripts are in the range $i, j=\{1,2\}$.

- The in-plane equilibrium equation:

$$
N_{i, j}+b_{i}=0
$$

Assuming linear elastic material eqns (1) and (2) can be written in terms of in-plane and out of plane displacements as follows:

$$
\begin{gathered}
D w_{i j i}=g+N_{i j} w_{i j} \quad(i, j=1,2) \\
\frac{1}{2(1-v)}\left(u_{j}, r_{i j}+w,_{j} w_{, i j}\right)+\frac{1}{2(1+v)}\left(u_{i}, r_{j j}+w,_{i} w,_{i j}\right)+b_{i} /(E h)
\end{gathered}
$$

where $D=E h^{3} /\left(1-v^{2}\right)$ is the flexural rigidity, $E$ and $v$ are the material Young modulus and Poisson's ratio.

The problem definition is then completed by assuming the following boundary conditions over $\Gamma: u_{i}=\bar{u}_{i}$ on $\Gamma_{l}$ (generalised displacements, in plane displacements, deflections and rotations) and $p_{i}=\bar{p}_{i}$ on $\Gamma_{2}$ (generalised tractions, in-plane tractions normal bending moment and effective shear forces), where $\Gamma_{1} \cup \Gamma_{2}=\Gamma$.

\section{Integral representations}

In this section, we are going to derive the integral equations of the plate bending and stretching problems considering geometrical non-linearities within the context previously defined. To obtain the integral equations of both problems one can apply the Betti's reciprocity to the linear parts of the stress and strain fields. Thus, for the bending problem the general reciprocity relation written for the $3 \mathrm{D}$ case can be integrated across the plate thickness to give:

$$
\int_{\Omega_{m}} w_{, i j}{ }_{i j} m_{i j} d \Omega=\int_{\Omega_{m}} m_{i j}^{*} w_{i_{i j}} d \Omega
$$

where $w^{*}$ and $m_{i j}^{*}$ are the well-known fundamental solutions of the plate problem given in terms of deflection and internal moments. These fundamental values and the other resulting required values are given in the specialized literature [8].

By integrating eqn (5) by parts twice and replacing the second derivative of the internal, $m_{i j}, i j$, according to eqn (1) one has: 


$$
\begin{aligned}
& C(S) w(S)+\int_{\Gamma}\left(V_{n}^{*}(S, P) w(P)-M_{n}^{*}(S, P) w_{n}(P)\right) d \Gamma(P)+\sum_{i=1}^{N_{c}} R_{c i}^{*}(S, P) w_{c i}(P) \\
& =\int_{\Gamma}\left(V_{n}(P) w^{*}(S, P)-M_{n}(P) w_{n}^{*}(S, P)\right) d \Gamma(P)+\sum_{i=1}^{N_{c}} R_{c i}(P) w_{c i}^{*}(S, P)+ \\
& \int_{\Omega_{g}} g(p) w^{*}(S, p) d \Omega(p)+\int_{\Omega} N_{i j}(p) w_{, i j}(p) w^{*}(S, p) d \Omega
\end{aligned}
$$

where $V_{n}$ and $M_{n}$ are effective shear forces, and moments applied along the boundary, respectively, $R_{c i}$ represents the corner reactions, $V_{n}^{*}, M_{n}^{*}$ and $R_{c i}^{*}$ the corresponding values obtained from the fundamental solution $w^{*}$ according to their definition.

For any internal collocation s one can differentiate eqn (6) to obtain the integral representations of rotations and curvatures, as follows:

$$
\begin{aligned}
& w,_{i}(s)=-\int_{\Gamma}\left\{V_{n}^{*},,_{i}(s, P) w(P)-M_{n}^{*},,_{i}(s, P) w,_{n}(P)\right\} d \Gamma(P)-\sum_{k=1}^{N_{c}} R_{c k}^{*},_{i}(s, P) w_{c k}(P) \\
& +\int_{\Gamma}\left\{V_{n}(P) w_{i_{i}}^{*}(q, P)-M_{n}(P) w_{,_{n i}}^{*}(s, P)\right\} d \Gamma(P)+\sum_{k=1}^{N_{c}} R_{c k}(P) w_{c k}^{*},_{i}(s, P)+ \\
& +\int_{\Omega_{g}} g(p) w_{i_{i}}^{*}(s, p) d \Omega(p)+\int_{\Omega} N_{j k}(p) w_{, j k}(p) w_{{ }_{i}}^{*}(s, p) d \Omega(p) \\
& w_{i_{i j}}(s)=-\int_{\Gamma}\left\{V_{n}^{*},_{i j}(s, P) w(P)-M_{n}^{*}, i_{i j}(s, P) w_{,_{n}}(P)\right\} d \Gamma(P)-\sum_{k=1}^{N_{c}} R_{c}^{*},,_{i j}(s, P) w_{c}(P) \\
& +\int_{\Gamma}\left\{V_{n}(P) w_{i j}^{*}(s, P)-M_{n}(P) w_{n i j}^{*}(s, P)\right\} d \Gamma(P)+\sum_{k=1}^{N_{c}} R_{c}(P) w_{c}^{*},_{i j}(s, P)+ \\
& +\int_{\Omega_{g}} g(p) w_{, j}^{*}(s, p) d \Omega(p)+\int_{\Omega} N_{k m}(p) w_{, k m}(p) w_{, j}^{*}(s, p) d \Omega(p)
\end{aligned}
$$

Analogously, the Betti's reciprocity relation for can be in applied to the linear parts of the 2D stretching problem to give:

$$
\int_{\Omega} N_{i j}^{\ell} \varepsilon_{i j}^{*} d \Omega=\int_{\Omega} N_{i j}^{*} \varepsilon_{i j} d \Omega
$$

where $N_{i j}^{\ell}$ represents the linear parts of the stretching problem internal forces, therefore given by:

$$
N_{i j}^{\ell}=\frac{E h}{(1-v)^{2}}\left[v u_{k},{ }_{k} \delta_{i j}+\frac{1-v}{2}\left(u_{i},{ }_{j}+u_{j},{ }_{i}\right)\right]
$$

After replacing $N_{i j}^{\ell}$ in eqn (13) and integrating it by parts the following integral representation is obtained for the in-plane boundary displacements: 


$$
\begin{gathered}
C_{i j}(S) u_{j}(S)=-\int_{\Gamma} P_{i j}^{*}(S, P) u_{j}(P) d \Gamma+\int_{\Gamma} u_{i j}^{*}(S, P) p_{j}(P) d \Gamma+ \\
+\int_{\Omega} u_{i j}^{*}(S, p) b_{j}(p) d \Omega-\frac{1}{2} \int_{\Omega} N_{i j k}^{*}(S, p) w_{, j}(p) w_{, k}(p) d \Omega
\end{gathered}
$$

By differentiating eqn (11) and then applying the Hooke's law at the collocation point $s$ accordingly, one has:

$$
\begin{aligned}
& N_{i j}(s)=\int_{\Gamma} D_{i j k}(s, P) p_{k}(P) d \Gamma-\int_{\Gamma} S_{i j k}(s, P) u_{k}(P) d \Gamma+\int_{\Omega} D_{i j k}(s, p) b_{k}(p) d \Omega \\
& -\frac{1}{2} \int_{\Omega} T_{i j k l}(s, p) w_{, k}(p) w_{, l}(p) d \Omega+\frac{G h}{8(1-\bar{v})}\left(2 w_{, i}(s) w_{, j}(s)+w_{, k}(s) w_{, k}(s) \delta_{i j}\right)
\end{aligned}
$$

where $\bar{v}=v /(1+v)$ is used to simulate the plane stress conditions.

\section{Algebraic equations}

Before transforming the integral representations derived in the previous section to algebraic equations let us replace the rate values by the corresponding increments. Let $\Delta t=t_{n+1}-t_{n}$ be a typical time-step in the time discretization. Any rate quantity $\dot{x}$ integrated along the time interval $\Delta t$ becomes $\Delta x=x_{n+1}-x_{n}$ that will replace $\dot{x}$ in all integral representations already derived.

As usual for any BEM formulation, the integral representations (6), (7), (8), (11) and (12) have to be transformed into algebraic expressions after discretizing the boundary and the domain. The plate boundary $\Gamma$ is then discretized into elements, $\Gamma_{s}$, along which generalized displacements and tractions are approximated using continuous and discontinuous linear boundary elements. In plate stretching-bending problems discontinuities are always present, particularly at corners and traction jumps. The discontinuity is always introduced by defining the collocation along the element or at any outside point near the boundary. Before transforming the integral representations, we decided replacing the density of the domain integrals into single values. The domain value $N_{i j}(p) w_{, i j}(p)$ at a field point $p$ will be replaced by a scalar value $T(p)$. Similarly, for the stretching problem, we replaced the domain value $w,{ }_{j}(p) w_{, k}(p)$ by domain tensor $W_{j k}(p)$. The approximation over the cells are now applied to these new domain values $T(p)$ and $W_{j k}(p)$. Triangular internal cells with linear shape functions are used with nodes always defined at internal points. Thus, discontinuous cells are required for cells adjacent to the boundary. The cell integrals are first transformed to integral over their sides and carried out as boundary elements using either analytical or appropriate numerical integration scheme with sub-elementation [9].

Carrying out the boundary and cell integrals eqn (6) written for all boundary nodes leads to the following incremental matrix equation: 


$$
\boldsymbol{H}_{b}^{w} \Delta \boldsymbol{U}^{b}=\boldsymbol{G}_{b}^{w} \Delta \boldsymbol{P}^{b}+\boldsymbol{S}_{b}^{w} \Delta \boldsymbol{T}_{N}^{\chi}+\Delta \boldsymbol{B}_{b}^{w}
$$

where $\Delta \boldsymbol{U}^{b}$ and $\Delta \boldsymbol{P}^{b}$ contains all boundary displacement and traction nodal values of the bending problem plus the additional corner displacements and reactions, $\Delta \boldsymbol{T}_{N}^{\chi}$ represents the condensate summation of the in-plane forces multiplied by curvatures, $\boldsymbol{S}_{b}^{w}$ is the corresponding matrices obtained by integrating all domain cells and $\Delta \boldsymbol{B}_{b}^{w}$ gives the domain load effects.

Similarly one can also transform the in-plane displacement integral representation, equation (7), into its incremental matrix form:

$$
\boldsymbol{H}_{s}^{u} \Delta \boldsymbol{U}^{s}=\boldsymbol{G}_{s}^{u} \Delta \boldsymbol{P}^{s}+\boldsymbol{S}_{s}^{u} \Delta \boldsymbol{W}_{\theta}^{\theta}+\Delta \boldsymbol{B}_{s}^{u}
$$

where $\Delta \boldsymbol{U}^{s}$ and $\Delta \boldsymbol{P}^{s}$ contains all incremental boundary displacement and traction nodal values of the stretching problem, $\Delta \boldsymbol{W}_{\theta}^{\theta}$ represents the increment of the rotation product defined at each domain node, $\boldsymbol{S}_{s}^{u}$ is the corresponding matrices obtained by integrating all domain cells and $\Delta \boldsymbol{B}_{s}^{u}$ gives the in-plane domain incremental load effects.

To complete the necessary algebraic relations one has to obtain the algebraic forms of the integral equations (7), (8) and (12), as follows:

$$
\begin{gathered}
\Delta \boldsymbol{\theta}=-\boldsymbol{H}_{b}^{\theta} \Delta \boldsymbol{U}^{b}+\boldsymbol{G}_{b}^{\theta} \Delta \boldsymbol{P}^{b}+\boldsymbol{S}_{b}^{\theta} \Delta \boldsymbol{T}_{N}^{\chi}+\Delta \boldsymbol{B}_{b}^{\theta} \\
\Delta \chi=-\boldsymbol{H}_{b}^{\chi} \Delta \boldsymbol{U}^{b}+\boldsymbol{G}_{b}^{\chi} \Delta \boldsymbol{P}^{b}+\boldsymbol{S}_{b}^{\chi} \Delta \boldsymbol{T}_{N}^{\chi}+\Delta \boldsymbol{B}_{b}^{\chi} \\
\Delta \boldsymbol{N}=-\boldsymbol{H}_{s}^{N} \Delta \boldsymbol{U}^{s}+\boldsymbol{G}_{s}^{N} \Delta \boldsymbol{P}^{s}+\boldsymbol{S}_{s}^{N} \Delta \boldsymbol{W}_{\theta}^{\theta}+\Delta \boldsymbol{B}_{s}^{N}
\end{gathered}
$$

where $\Delta \boldsymbol{\theta}, \Delta \chi$ and $\Delta \boldsymbol{N}$ are vectors containing rotation, curvature and membrane internal force increments at the domain nodes defined by the adopted discretization.

After applying the boundary conditions equations (13), (14) and (15)-(17) become:

$$
\begin{gathered}
\boldsymbol{A}_{b}^{w} \Delta \boldsymbol{X}^{b}=\Delta \boldsymbol{F}_{b}^{w}+\boldsymbol{S}_{b}^{w} \Delta \boldsymbol{T}_{N}^{\chi} \\
\boldsymbol{A}_{s}^{u} \Delta \boldsymbol{X}^{s}=\Delta \boldsymbol{F}_{s}^{u}+\boldsymbol{S}_{s}^{u} \Delta \boldsymbol{W}_{\theta}^{\theta} \\
\Delta \boldsymbol{\theta}=-\boldsymbol{A}_{b}^{\theta} \Delta \boldsymbol{X}^{b}+\Delta \boldsymbol{F}_{b}^{\theta}+\boldsymbol{S}_{b}^{\theta} \Delta \boldsymbol{T}_{N}^{\chi} \\
\Delta \chi=-\boldsymbol{A}_{b}^{\chi} \Delta \boldsymbol{X}^{b}+\Delta \boldsymbol{F}_{b}^{\chi}+\boldsymbol{S}_{b}^{\chi} \Delta \boldsymbol{T}_{N}^{\chi} \\
\Delta \boldsymbol{N}=-\boldsymbol{A}_{s}^{N} \Delta \boldsymbol{U}^{b}+\Delta \boldsymbol{F}_{s}^{N}+\boldsymbol{S}_{s}^{N} \Delta \boldsymbol{W}_{\theta}^{\theta}
\end{gathered}
$$

where the matrices $\boldsymbol{A}_{x}^{y}$ are conveniently built up using columns of $\boldsymbol{H}_{x}^{y}$ and $\boldsymbol{G}_{x}^{y}$ corresponding to unknown boundary values, and $\Delta \boldsymbol{F}_{x}^{y}$ is a vector collecting all contributions of prescribed domain and boundary values. 
Solving eqns (18) and (19) and replacing into eqns (20) - (22) gives:

$$
\begin{gathered}
\Delta \boldsymbol{\theta}=\Delta \boldsymbol{N}_{b}^{\theta}+\boldsymbol{Q}_{b}^{\theta} \Delta \boldsymbol{T}_{N}^{\chi} \\
\Delta \chi=\Delta \boldsymbol{N}_{b}^{\chi}+\boldsymbol{Q}_{b}^{\chi} \Delta \boldsymbol{T}_{N}^{\chi} \\
\Delta \boldsymbol{N}=\Delta \boldsymbol{N}_{s}^{N}+\boldsymbol{Q}_{s}^{N} \Delta \boldsymbol{W}_{\theta}^{\theta}
\end{gathered}
$$

where $\quad \Delta \boldsymbol{N}_{x}^{y}=-\boldsymbol{A}_{x}^{y} \Delta \boldsymbol{M}_{x}^{z}+\Delta \boldsymbol{F}_{x}^{y} \quad$ and $\quad \boldsymbol{Q}_{x}^{y}=-\boldsymbol{A}_{x}^{y} \boldsymbol{Q}_{x}^{z}+\boldsymbol{S}_{x}^{y}, \quad$ with $\Delta \boldsymbol{M}_{x}^{z}=\left[\boldsymbol{A}_{x}^{z}\right]^{-1} \Delta \boldsymbol{F}_{x}^{z}$ and $\boldsymbol{Q}_{x}^{z}=\left[\boldsymbol{A}_{x}^{z}\right]^{-1} \boldsymbol{S}_{x}^{z}$ and $z$ given by the superscript of the corresponding boundary algebraic equation used to compute $\boldsymbol{Q}_{x}^{z}$, i.e. $\boldsymbol{Q}_{x}^{w}$ from eqn (18) or $\boldsymbol{Q}_{x}^{u}$ from eqn (19).

Equations (23) - (25) are the necessary relations to solve a geometrical nonlinear plate problem. However, one has to treat correctly the increments $\Delta W_{j k}(p)$ and $\Delta T(p)$. We can first find the rates of the densities in eqns (6) and (11) and obtain the incremental forms of $\Delta \boldsymbol{T}$ and $\Delta \boldsymbol{W}$ for a given time interval $\Delta t_{n}$, as follows:

$$
\begin{aligned}
& \Delta T=\Delta N \bullet \chi+N \bullet \Delta \chi \\
& \Delta W=\Delta \theta \otimes \theta+\theta \otimes \Delta \theta
\end{aligned}
$$

\section{System solution}

Equations (23) - (25) represent the non-linear system to be solved in terms of the increments $\Delta \boldsymbol{\theta}, \Delta \chi$ and $\Delta \boldsymbol{N}$. Replacing the increments $\Delta \boldsymbol{T}$ and $\Delta \boldsymbol{W}$ according to eqns (26) and (27) one has:

$$
\begin{gathered}
F_{\theta}(\Delta \boldsymbol{\theta}, \Delta \chi, \Delta \boldsymbol{N})=-\Delta \boldsymbol{\theta}+\Delta \boldsymbol{N}_{b}^{\theta}+Q_{b}^{\theta} \Delta \boldsymbol{N} \cdot \chi+Q_{b}^{\theta} \boldsymbol{N} \cdot \Delta \chi=0 \\
F_{\chi}(\Delta \boldsymbol{\theta}, \Delta \chi, \Delta \boldsymbol{N})=-\Delta \chi+\Delta \boldsymbol{N}_{b}^{\chi}+Q_{b}^{\chi} \Delta \boldsymbol{N} \cdot \chi+Q_{b}^{\chi} \boldsymbol{N} \cdot \Delta \chi \\
F_{N}(\Delta \boldsymbol{\theta}, \Delta \chi, \Delta \boldsymbol{N})=-\Delta \boldsymbol{N}+\Delta \boldsymbol{N}_{s}^{N}+Q_{s}^{N} \Delta \boldsymbol{\theta} \otimes \boldsymbol{\theta}+Q_{s}^{N} \boldsymbol{\theta} \otimes \Delta \boldsymbol{\theta}
\end{gathered}
$$

The above non-linear system of equations is solved by applying the NewtonRaphson's scheme. Within a time increment $\Delta t_{n}=t_{n+1}-t_{n}$ an iterative process may be required to achieve the equilibrium. Then, from the solution at iteration $i$ the next try at iteration $(i+1)$ is given by:

$$
\begin{gathered}
\left\{\Delta \boldsymbol{N}_{n}^{i+1}\right\}=\left\{\Delta \boldsymbol{N}_{n}^{i}\right\}+\left\{\delta \Delta \boldsymbol{N}_{n}^{i}\right\} \\
\left\{\Delta \boldsymbol{\theta}_{n}^{i+1}\right\}=\left\{\Delta \boldsymbol{\theta}_{n}^{i}\right\}+\left\{\delta \Delta \boldsymbol{\theta}_{n}^{i}\right\}
\end{gathered}
$$




$$
\left\{\Delta \chi_{n}^{i+1}\right\}=\left\{\Delta \chi_{n}^{i}\right\}+\left\{\delta \Delta \chi_{n}^{i}\right\}
$$

By linearizing eqns (28) - (30), using the first term of the Taylor's expansion, gives:

$$
\left\{\begin{array}{l}
F_{\theta}\left(\Delta \boldsymbol{\theta}_{n}^{i}, \Delta \chi_{n}^{i}, \Delta \boldsymbol{N}_{n}^{i}\right) \\
F_{\chi}\left(\Delta \boldsymbol{\theta}_{n}^{i}, \Delta \chi_{n}^{i}, \Delta \boldsymbol{N}_{n}^{i}\right) \\
F_{N}\left(\Delta \boldsymbol{\theta}_{n}^{i}, \Delta \chi_{n}^{i}, \Delta \boldsymbol{N}_{n}^{i}\right)
\end{array}\right\}+\left[\begin{array}{ccc}
\frac{\partial F_{\theta}}{\partial \Delta \boldsymbol{\theta}_{n}^{i}} & \frac{\partial F_{\theta}}{\partial \Delta \chi_{n}^{i}} & \frac{\partial F_{\theta}}{\partial \Delta \boldsymbol{N}_{n}^{i}} \\
\frac{\partial F_{\chi}}{\partial \Delta \boldsymbol{\theta}_{n}^{i}} & \frac{\partial F_{\chi}}{\partial \Delta \chi_{n}^{i}} & \frac{\partial F_{\chi}}{\partial \Delta \boldsymbol{N}_{n}^{i}} \\
\frac{\partial F_{N}}{\partial \Delta \boldsymbol{\theta}_{n}^{i}} & \frac{\partial F_{N}}{\partial \Delta \chi_{n}^{i}} & \frac{\partial F_{N}}{\partial \Delta \boldsymbol{N}_{n}^{i}}
\end{array}\right]\left\{\begin{array}{l}
\delta \Delta \boldsymbol{\theta}_{n}^{i} \\
\delta \Delta \chi_{n}^{i} \\
\delta \Delta \boldsymbol{N}_{n}^{i}
\end{array}\right\}+\ldots=0
$$

where each term of the tangent matrix is given by:

$$
[C]=\left[\begin{array}{ccc}
-\boldsymbol{I} & \boldsymbol{Q}_{b}^{\theta} \boldsymbol{N}_{n}^{i} \bullet \boldsymbol{I I} & \boldsymbol{Q}_{b}^{\theta} \boldsymbol{I I} \bullet \chi_{n}^{i} \\
\boldsymbol{0} & -\boldsymbol{I I}+\boldsymbol{Q}_{b}^{\chi} \boldsymbol{N}_{n}^{i} \bullet \boldsymbol{I I} & \boldsymbol{Q}_{b}^{\chi} \boldsymbol{I} \bullet \chi_{n}^{i} \\
\boldsymbol{Q}_{s}^{N}\left(\boldsymbol{I} \otimes \boldsymbol{\theta}_{n}^{i}+\boldsymbol{\theta}_{n}^{i} \otimes \boldsymbol{I}\right) & \boldsymbol{0} & -\boldsymbol{I I}
\end{array}\right]
$$

Thus, the corrections to be cumulated during the iterations are obtained by solving eqn (34), as follows:

$$
\left\{\begin{array}{l}
\delta \Delta \boldsymbol{\theta}_{n}^{i} \\
\delta \Delta \chi_{n}^{i} \\
\delta \Delta \boldsymbol{N}_{n}^{i}
\end{array}\right\}=-[\boldsymbol{C}]^{-1}\left\{\begin{array}{l}
F_{\theta}\left(\Delta \theta_{n}^{i}, \Delta \chi_{n}^{i}, \Delta \boldsymbol{N}_{n}^{i}\right) \\
F_{\chi}\left(\Delta \boldsymbol{\theta}_{n}^{i}, \Delta \chi_{n}^{i}, \Delta \boldsymbol{N}_{n}^{i}\right) \\
F_{N}\left(\Delta \boldsymbol{\theta}_{n}^{i}, \Delta \chi_{n}^{i}, \Delta \boldsymbol{N}_{n}^{i}\right)
\end{array}\right\}
$$

\section{Numerical application}

To check the performance of the proposed formulation we have chosen a square plate subjected to a uniform load (figure 2). The plate side length is $a$, the thickness is $t$, with the ration $t / a=0.0 .1$. The Poisson's ration was assumed $v=0.3$ while $q$ is the uniform applied load.

Several boundary conditions have been analyzed. We started by assuming simple supported and clamped plate conditions. For each of this case the in-plane boundary displacements could also be prescribed equal to zero (IE) or kept free (ME).

The results obtained are shown in figures 3 and 4, for the simply supported plate and clamped plate respectively. As can be seen the results are in total agreement with the one given by Ye and Liu [3].

The obtained results, compared with other numerical and analytical solutions demonstrated that the proposed formulation is accurate. Running several other internal and boundary meshes has also demonstrated that the convergence is obtained quickly. With a rather coarse mesh, particularly to integrate the domain integrals, the results are already accurate. The presented results were obtained by using a 160 boundary elements and only 8 domain cells 
Boundary Elements and Other Mesh Reduction Methods XXIX 221

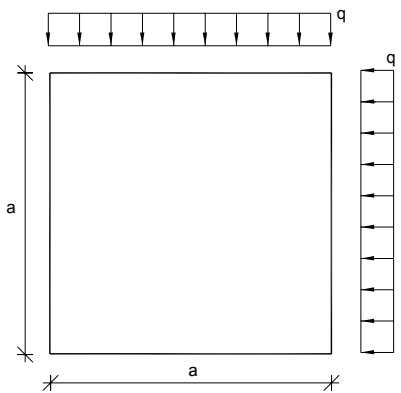

Figure 2: $\quad$ Square plate with uniform load.

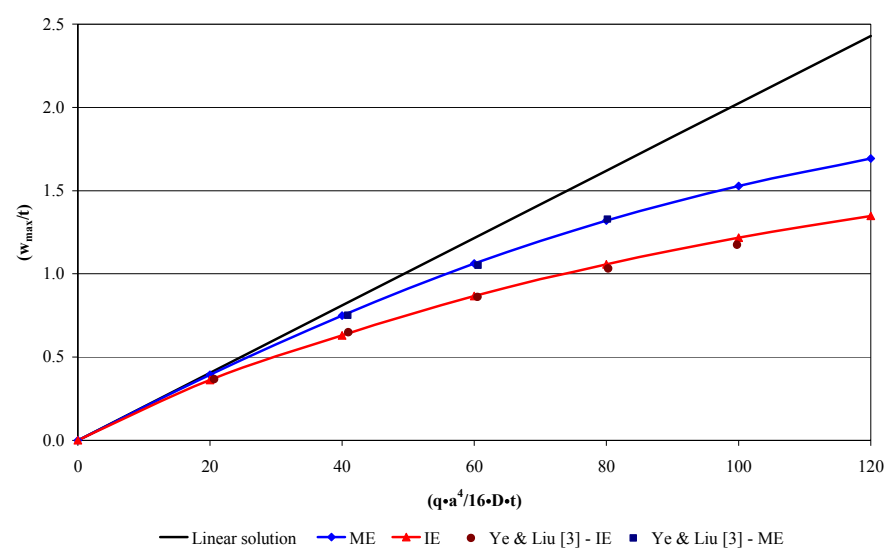

Figure 3: $\quad$ Simply supported plate - load displacement curve.

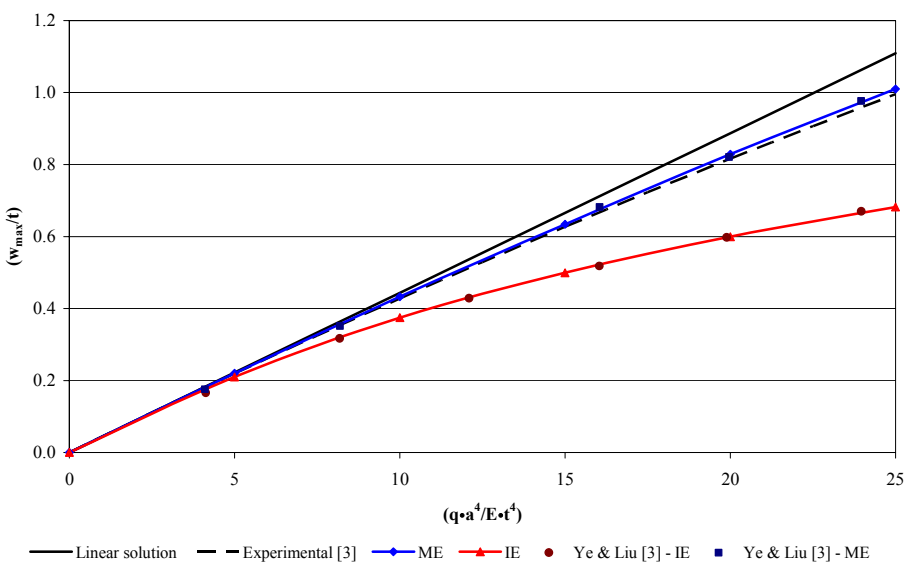

Figure 4: $\quad$ Clamped plate - load displacement curve. 


\section{Conclusions}

A boundary element formulation to analyse von Kármán plates was proposed. The domain approximations were simplified by reducing the density of domain integrals into simple equivalent values reducing the computational effort to compute the matrices related with the domain values. A consistent tangent operator has been derived and the Newton process has been implement leading accurate and reliable solutions using a very small number of iterations.

\section{Acknowledgments}

To FAPESP - São Paulo State Research Foundation for the support given to this work.

\section{References}

[1] Morjaria, M., Inelastic analysis of transverse deflection of plates by the boundary element method. Journal of Applied Mechanics-Transactions of the ASME, 47: 291-, 1980

[2] Kamiya, N. \& Sawaki, Y., An integral equation approach to finite deflection of elastic plates. International Journal of Non-Linear Mechanics, 17(3): 187-194, 1982.

[3] Ye, T.-Q. \& Liu, Y.-J., Finite deflection analysis of elastic plate by the boundary element method. Applied Mathematical Modelling, 9: 183-188, 1985.

[4] Tanaka, M., Matsumoto, T. \& Zheng, Z.-D. Incremental analysis of finite deflection of elastic plates via boundary-domain-element method. Engineering Analysis with Boundary Elements, 17: 123-131, 1996.

[5] Wang, W., Ji, X. \& Tanaka, M., A dual boundary element approach for the problems of large deflection of thin elastic plates. Computational Mechanics, 26: 58-65, 2000.

[6] Wen, P.H., Aliabadi, M.H. \& Young, A., Large deflection analysis of Reissner plate by boundary element method. Computers \& Structures, 83 (10-11): 870-879, 2005.

[7] Purbolaksono, J. \& Aliabadi, M.H., Large deformation of shear-deformable plates by the boundary element method. Journal of Engineering Mathematics 51 (3): 211-230, 2005.

[8] Brebbia, C.A., Telles, J.C.F \& Wrobel, L.C. Boundary Element Techniques. Theory and Applications in Engineering, Springer-Verlag: Berlin and New York, 1984.

[9] Leite, L.G.S. \& Venturini, W.S., Stiff and soft thin inclusions in twodimensional solids by the boundary element method. Engineering Analysis with Boundary Elements, 29 (3): 257-267, 2005. 